\title{
On the MIMO Channel Capacity of Multi-Dimensional Signal Sets
}

\author{
S. X. Ng and L. Hanzo \\ School of ECS, University of Southampton, SO17 1BJ, UK. \\ Tel: +44-23-8059 3125, Fax: +44-23-8059 4508 \\ Email: $\{$ sxn,lh\}@ecs.soton.ac.uk, http://www-mobile.ecs.soton.ac.uk
}

\begin{abstract}
In this contribution two general formulae were derived for the capacity evaluation of Multi-Input Multi-Output (MIMO) systems using multi-dimensional signal sets, different modulation schemes and an arbitrary number of transmit as well as receive antennas. It was shown that transmit diversity is capable of narrowing the gap between the capacity of the Rayleighfading channel and the AWGN channel. However, since this gap becomes narrower when the receiver diversity order is increased, for higher-order receiver diversity the performance advantage of transmit diversity diminishes. A MIMO system having full multiplexing gain has a higher achievable capacity, than the corresponding MIMO system designed for achieving full diversity gain, provided that the channel SNR is sufficiently high.
\end{abstract}

\section{INTRODUCTION}

The capacity, $C$, of a Single-Input Single-Output (SISO) AWGN channel was quantified by Shannon in 1948 [1]. Since then, substantial research efforts have been invested in finding channel codes that would produce an arbitrarily low probability of error at a transmission rate close to $C^{*}=C / T$, where $T$ is the symbol period. We note however that Shannon's channel capacity is only defined for ContinuousInput Continuous-Output Memoryless Channels (CCMC) [2], where the channel input is a continuous-amplitude, discrete-time Gaussiandistributed signal and the capacity is only restricted either by the signalling energy or by the bandwidth. Therefore we will refer to the capacity of the CCMC as the unrestricted bound.

By contrast, in the context of discrete-amplitude QAM and PSK [2] signals, we encounter a Discrete-Input Continuous-Output Memoryless Channel (DCMC) [2]. Therefore, the capacity of the DCMC is more pertinent in the design of channel coded modulation schemes. With the advent of powerful space-time coding schemes [3, 4], the Multi-Input Multi-Output (MIMO) channel capacity is of immediate interest. Note that multiple antennas can be utilised for providing diversity gain and/or multiplexing gain [5]. Specifically, Space-Time Trellis Coding (STTC) [6] and Space-Time Block Coding (STBC) $[7,8]$ were designed for achieving diversity gains by conveying the same information through different paths over the MIMO channel in order to combat the channel-induced fading. By contrast, Bell Lab's Layered Space-Time (BLAST) [9] scheme transmits independent information in parallel over the MIMO channel for the sake of achieving multiplexing gain, hence increasing the attainable transmission rate. Furthermore, both STTC and STBC schemes are capable of achieving full transmit diversity ${ }^{1}$ at the cost of providing no multiplexing gain, while the BLAST scheme was designed for achieving full multiplexing gain at the cost of having no transmit diversity gain. The tradeoffs associated with having partial diversity gain and partial multiplexing

\footnotetext{
${ }^{1}$ A system is said to have a full transmit diversity, when the transmit diversity order is identical to the number of transmit antennas [7].
}

gain when communicating over MIMO channels was studied in [5]

However, the MIMO channel's capacity was only found for the CCMC in [3]. Furthermore, only the SISO AWGN channel capacity was found for multi-dimensional signal sets, such as $M$-ary orthogonal signalling [2] and $L$-ary PSK based $L$-orthogonal signalling [10, 11]. More specifically, the $L$-orthogonal PSK signal [11] is a hybrid form of $M$-ary orthogonal and PSK signalling, combining the benefits of power-efficient and error-resilient $M$-ary orthogonal signalling [2, p. 284] as well as bandwidth-efficient PSK signalling. At this stage we note that STTC and STBC schemes have so far been exclusively designed for complex-valued (two-dimensional) PSK/QAM signal sets, but not for multi-dimensional signal sets. Against this background, the novel contribution of this treatise is that we provide two general channel capacity formulae applicable to MIMO channels exhibiting full diversity gain or full multiplexing gain as well as employing multi-dimensional signal sets, in the quest for more error-resilient, power-efficient and bandwidth-efficient MIMO channel coding schemes.

\section{MULTIDIMENSIONAL SIGNAL SET}

The dimensionality of a time- and band-limited signal is defined as [12, pp. 348-351] $]^{2}: D=2 W T$, where $W$ is the bandwidth and $T$ is the signalling period of the finite-energy signalling waveform. In an $L$ orthogonal PSK signal set $[10,11]$, there are $V=W T$ independent $L$-ary PSK subsets. The total number of waveforms is $M=V L$ and the number of dimensions is $D=2 \mathrm{~V}$, which is independent of $L$. Specifically, an $L$-orthogonal PSK signal requires splitting the original PSK symbol period into $V$ number of proportionately shortened PSK symbol periods and hence necessitates $V$ times the bandwidth of PSK signalling, in order to transmit $\log _{2}(M)$ bits. The vector representation of $L$-orthogonal PSK signalling may be formulated as:

$$
\mathbf{x}_{m}=\mathbf{x}_{l}^{L P S K} \phi_{v}, m=1, \ldots, M, l=m \% L,
$$

where $m \% L$ is the remainder of $m / L$, while $v=\left(\frac{m-l}{V}+1\right)$ and $\mathbf{x}_{l}^{L P S K}$ is the classic 2-dimensional $L$-ary PSK signal vector. Furthermore, the orthonormal basis function $\phi_{v}=\left(\phi_{v}[1], \phi_{v}[2], \ldots, \phi_{v}[V]\right)$ is a vector of $V$ elements, which may be constructed from non-overlapping signalling pulses as follows:

$$
\phi_{m}[i]= \begin{cases}1, & i=m \\ 0, & i \neq m .\end{cases}
$$

Figure 1 illustrates an example of $L=8$-orthogonal PSK signalling having $V=2$. For $V=1, L$-orthogonal PSK signalling repre-

\footnotetext{
${ }^{2}$ This dimensionality is different from the definition used in the context of multidimensional trellis coded modulation [13] (MTCM). Explicitly, when the MTCM-dimensionality increases, only the coding rate increases, but the corresponding capacity and bandwidth efficiency curves representing the achievable upper-bound performance of the PSK/QAM signalling remain the same.
} 
$T_{s}=2 T_{p}$
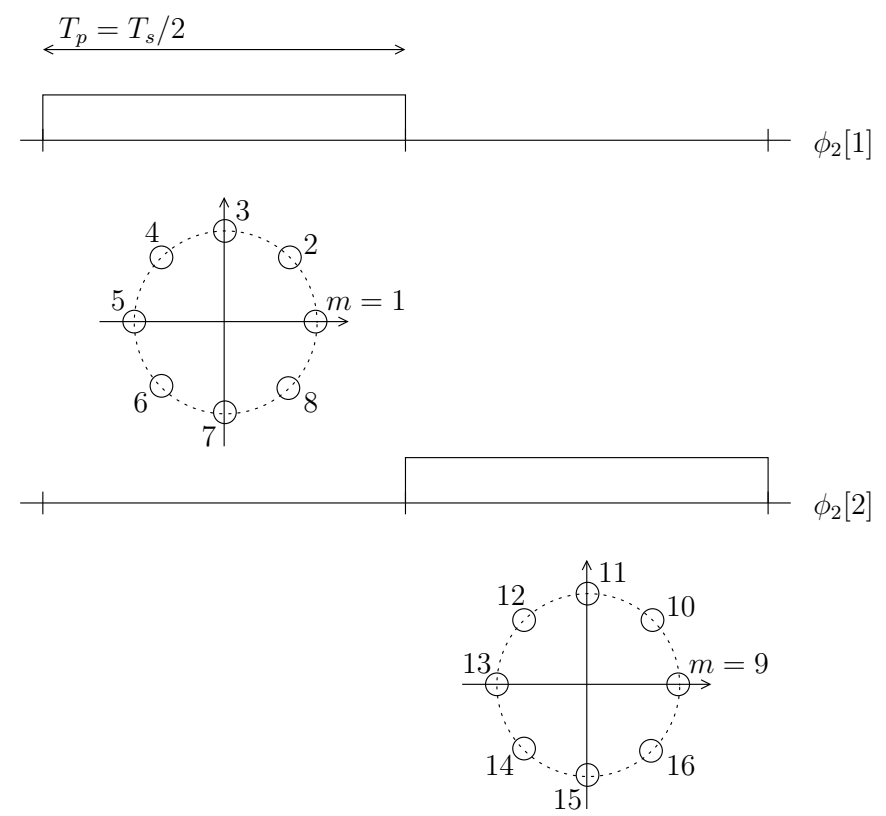

Figure 1: An $L$-orthogonal PSK example conveying 4 bits per symbol using $L=8$-ary PSK subset, where the total symbol period $T_{s}$ consists of $V=2$ LPSK subset's signalling durations $T_{p}$.

sents classic two-dimensional $L$-ary PSK signalling. The total number of waveforms is $M=V L=16$ and the number of dimensions is $D=2 V=4$. Note that only one timeslot of duration $T_{p}$ is active during the symbol period of $T_{s}=V T_{p}$. Therefore, $L$-orthogonal PSK signalling achieves $\log _{2}(V)$ bits higher capacity at the cost of $V$ times lower bandwidth efficiency, than that of classic $L$-ary PSK signalling. As a further contribution to the current state-of-the-art in the literature, we found that the concept of L-orthogonal PSK signalling can also be extended to QAM, rather than being limited to PSK.

\section{MIMO CHANNEL CAPACITY BOUND FOR FULL DIVERSITY GAIN}

When $D=2$-dimensional PSK/QAM was employed, the received signal of Alamouti's orthogonal STBC [7] having $N_{t}=2$ transmit antennas and $N_{r}$ receive antennas can be transformed into [14]:

$$
\mathbf{y}=\sum_{n=1}^{N}\left|\mathbf{h}_{n}\right|^{2} \mathbf{x}+\boldsymbol{\Omega}=\chi_{2 N}^{2} \mathbf{x}+\boldsymbol{\Omega},
$$

where $\mathbf{y}, \mathbf{x}$ and $\mathbf{h}_{n}$ are the complex-valued transformed received signal, the complex-valued transmitted signal and the complex-valued Rayleigh-fading coefficient of the $n$th MIMO channel link, respectively. Furthermore, $\chi_{2 N}^{2}=\sum_{n=1}^{N}\left|\mathbf{h}_{n}\right|^{2}$ represents the chi-squared distributed random variable having $2 N$ degree of freedom, where we have $N=N_{t} \times N_{r}$. Finally, $\boldsymbol{\Omega}$ is the MIMO system's complexvalued AWGN after transformation, which has a zero mean and a variance of $\chi_{2 N}^{2} N_{0} / 2$ per dimension, where $N_{0} / 2$ is the original noise's variance per dimension. It was shown in [15] that a full-rate, fulldiversity orthogonal STBC also exists for $N_{t}>2$. Let us now generalise Equation 3 for a $D>2$-dimensional $L$-orthogonal PSK/QAM scheme as:

$$
\begin{aligned}
\mathbf{y} & =\sum_{d=1}^{D}\left(\chi_{2 N}^{2}[d] x[d]+\Omega[d]\right), \\
y[d] & =\chi_{2 N}^{2}[d] x[d]+\Omega[d],
\end{aligned}
$$

where $\mathbf{y}=(y[1], \ldots, y[D]), \mathbf{x}=(x[1], \ldots, x[D])$ and $\boldsymbol{\Omega}=(\Omega[1], \ldots, \Omega[D])$. Note that when $D>2$, we have $D / 2$ number of different $\chi_{2 N}^{2}$ values for the $D$-dimensional signals. Specifically, we have $\chi_{2 N}^{2}[i]=\chi_{2 N}^{2}[i+1]$ for $i \in\{1,3,5 \ldots\}$, since a complex channel has two dimensions. Furthermore, $\Omega[d]$ has a variance of $\chi_{2 N}^{2}[d] N_{0} / 2$ per each $D$ dimensions.

The conditional probability of receiving a $D$-dimensional signal $\mathbf{y}$ given that a $D$-dimensional $M$-ary signal $\mathbf{x}_{m}, m \in\{1, \ldots, M\}$, was transmitted over an AWGN channel is determined by the PDF of the noise, yielding:

$$
p\left(\mathbf{y} \mid \mathbf{x}_{m}\right)=\prod_{d=1}^{D} \frac{1}{\sqrt{\pi N_{0}}} \exp \left(\frac{-\left(y[d]-x_{m}[d]\right)^{2}}{N_{0}}\right)
$$

where $N_{0} / 2$ is the channel's noise variance. For the full-diversity MIMO system of Equation 5, we have:

$$
\begin{aligned}
p\left(\mathbf{y} \mid \mathbf{x}_{m}\right)= & \frac{1}{\prod_{d=1}^{D} \sqrt{\pi \chi_{2 N}^{2}[d] N_{0}}} \cdot \\
& \exp \left(\sum_{d=1}^{D} \frac{-\left(y[d]-\chi_{2 N}^{2}[d] x_{m}[d]\right)^{2}}{\chi_{2 N}^{2}[d] N_{0}}\right) .
\end{aligned}
$$

The channel capacity for the $N=\left(N_{t} \times N_{r}\right)$ MIMO using $D$ dimensional $M$-ary signalling over the DCMC can be derived from that of the Discrete Memoryless Channel (DMC) [16] as:

$$
\begin{aligned}
C_{\mathrm{DCMC}}^{N}= & \max _{p\left(\mathbf{x}_{1}\right) \ldots p\left(\mathbf{x}_{M}\right)} \sum_{m=1}^{M} \int_{-\infty}^{\infty} \ldots \int_{-\infty}^{\infty} p\left(\mathbf{y} \mid \mathbf{x}_{m}\right) p\left(\mathbf{x}_{m}\right) . \\
& \log _{2}\left(\frac{p\left(\mathbf{y} \mid \mathbf{x}_{m}\right)}{\sum_{i=1}^{M} p\left(\mathbf{y} \mid \mathbf{x}_{i}\right) p\left(\mathbf{x}_{m}\right)}\right) d \mathbf{y}[\mathrm{bit} / \mathrm{sym}],
\end{aligned}
$$

where $p\left(\mathbf{x}_{m}\right)$ is the probability of occurrence for the transmitted signal $\mathbf{x}_{m}$. We know that Equation 8 is maximised, when the transmitted signals are equiprobably distributed, i.e. when we have $p\left(\mathbf{x}_{m}\right)=$ $1 / M$. Hence, we have:

$$
\begin{aligned}
\log _{2}\left(\frac{p\left(\mathbf{y} \mid \mathbf{x}_{m}\right)}{\sum_{i=1}^{M} p\left(\mathbf{y} \mid \mathbf{x}_{i}\right) p\left(\mathbf{x}_{m}\right)}\right)= & -\log _{2}\left(\frac{1}{M} \sum_{i=1}^{M} \frac{p\left(\mathbf{y} \mid \mathbf{x}_{i}\right)}{p\left(\mathbf{y} \mid \mathbf{x}_{m}\right)}\right) \\
= & \log _{2}(M)- \\
& \log _{2} \sum_{i=1}^{M} \exp \left(\Phi_{i}^{m}(N)\right)
\end{aligned}
$$

which is dependent on:

$$
\begin{aligned}
\Phi_{i}^{m}(N) & =\sum_{d=1}^{D} \frac{-\left(y[d]-\chi_{2 N}^{2}[d] x_{i}[d]\right)^{2}+\left(y[d]-\chi_{2 N}^{2}[d] x_{m}[d]\right)^{2}}{\chi_{2 N}^{2}[d] N_{0}} \\
& =\sum_{d=1}^{D} \frac{-\left(\chi_{2 N}^{2}[d]\left(x_{m}[d]-x_{i}[d]\right)+\Omega[d]\right)^{2}+\Omega^{2}[d]}{\chi_{2 N}^{2}[d] N_{0}}
\end{aligned}
$$


By substituting Equation 9 and $p\left(\mathbf{x}_{m}\right)=1 / M$ into Equation 8 we have:

$$
\begin{aligned}
C_{\mathrm{DCMC}}^{N}= & \frac{\log _{2}(M)}{M} \sum_{m=1}^{M} \int_{-\infty}^{\infty} \ldots \int_{-\infty}^{\infty} p\left(\mathbf{y} \mid \mathbf{x}_{m}\right) d \mathbf{y}- \\
& \frac{1}{M} \sum_{m=1}^{M} \int_{-\infty}^{\infty} \ldots \int_{-\infty}^{\infty} p\left(\mathbf{y} \mid \mathbf{x}_{m}\right) \log _{2} \sum_{i=1}^{M} \exp \left(\Phi_{i}^{m}(N)\right) d \mathbf{y} \\
= & \log _{2}(M)-\text { D-fold } \\
& \frac{1}{M} \sum_{m=1}^{M} E\left[\log _{2} \sum_{i=1}^{M} \exp \left(\Phi_{i}^{m}(N)\right)\right][\mathrm{bit} / \mathrm{sym}]
\end{aligned}
$$

where the expectation in Equation 11 is taken over $\chi_{2 N}^{2}[d]$ and $\Omega[d]$. This expected value can be estimated using the Monte Carlo averaging method. More specifically, Equation 11 represents the capacity of the MIMO DCMC achieving full diversity gain for $D$-dimensional, $M$ ary QAM/PSK signals employing $N_{t}$ number of transmit antennas and $N_{r}$ number of receive antennas.

Note that in a SISO AWGN channel we have $\chi_{2 N}^{2}[d]=N=1$ and hence the noise variance of $\Omega[d]$ is $N_{0} / 2$ per each dimension. For $D=2$-dimensional signalling, Equation 10 can be simplified to: $\Phi_{i}^{m}=\frac{-\left|\chi_{2 N}^{2}\left(\mathbf{x}_{m}-\mathbf{x}_{i}\right)+\boldsymbol{\Omega}\right|^{2}+|\boldsymbol{\Omega}|^{2}}{\chi_{2 N}^{2} N_{0}}$, where $\mathbf{x}_{k}=x_{k}[1]+j x_{k}[2]$ and $\boldsymbol{\Omega}=\Omega[1]+j \Omega[2]$. It is reassuring to note that in the simplified case of SISO AWGN channels, Equations 10 and 11 agree with the results of [17]. The average SNR can be determined from $[10,17]$ as:

$$
S N R=\frac{\frac{1}{M} \sum_{m=1}^{M} \sum_{d=1}^{D}\left|x_{m}[d]\right|^{2}}{\sum_{d=1}^{D} \mathrm{E}\left[\Omega^{2}[d]\right]}=\frac{E_{s}}{D N_{0} / 2},
$$

where $E_{s}$ is the average energy of the $D$-dimensional $M$-ary symbol $\mathbf{x}_{m}$ and $D \frac{N_{0}}{2}$ is the average energy of the $D$-dimensional AWGN $\boldsymbol{\Omega}$. Additionally, the energy of the signal sets is further normalised by $\sqrt{N_{t}}$, when the transmitter does not know the complex Rayleighdistributed channel coefficient of each of the MIMO links. Hence, we have $x_{k}[d]=\tilde{x}_{k}[d] / \sqrt{N_{t}}$, where $\tilde{x}_{k}[d]$ is the $k$ th modulated signal, $k=\{1, \ldots, M\}$, of dimension $d$ in the case of $N_{t}=1$. In an AWGN channel, the channel capacity is not expected to increase, when $N_{t}$ is increased. However, if the transmitter knows the complex Rayleigh-distributed channel coefficient of each of the MIMO links, the transmitted power to be assigned to the various transmit antennas can be distributed according to the "water-filling" principle [3] in order to increase the achievable capacity. In this scenario, the energy of the signal sets does not have to be further normalised by $\sqrt{N_{t}}$. The capacity formula of Equations 10 and 11 can also be applied to real-valued signal sets, such as $M$-ary orthogonal signals, as well as to amplitude-modulated signals following straightforward adjustments of the signalling space dimensionality, the channel fading and the noise. The unrestricted MIMO CCMC capacity achieving full diversity gain can be derived based on [3] as:

$$
\begin{aligned}
C_{\mathrm{CCMC}}^{N} & =E\left[W T \log _{2}\left(1+\chi_{2 N}^{2} \frac{S N R}{N_{t}}\right)\right][\mathrm{bit} / \mathrm{sym}] \\
& =E\left[\frac{D}{2} \log _{2}\left(1+\chi_{2 N}^{2} \frac{S N R}{N_{t}}\right)\right][\mathrm{bit} / \mathrm{sym}]
\end{aligned}
$$

where the expectation is taken over $\chi_{2 N}^{2}$, when the transmitter does not know the channel. Again, the normalisation factor $N_{t}$ is dropped from the equation, when the transmitter knows the channel [3].

\section{MIMO CHANNEL CAPACITY BOUND FOR FULL MULTIPLEXING GAIN}

It was shown in [3] that MIMO channels employing $N_{t}$ number of transmit antennas and $N_{r}$ number of receive antennas can be considered as $r$ uncoupled parallel channels with the aid of the singular value decomposition method and a transformation on the received signal vector. Specifically, $r=\min \left(N_{t}, N_{r}\right)$ is the maximum achievable rank of the MIMO channel matrix. Since the subchannels are uncoupled, their capacities add up. Hence a full multiplexing gain is attained, when all $r$ number of uncoupled subchannels are used for conveying independent information. We define a full-multiplexinggain system as the MIMO system achieving full multiplexing gain without any transmit/receive diversity gain. Note that the Multiple Antenna Interference (MAI) encountered in the BLAST scheme can be utilised for achieving receive diversity gain at the cost of employing an interference cancellation scheme. By contrast, a MIMO system benefiting from orthogonal transmission [3, p. 11] is free from MAI and achieves no receive/transmit diversity gain.

Let us now quantify the capacity of the system having full multiplexing gain using a MIMO system benefiting from orthogonal transmission having $\mathcal{N}=N_{t}=N_{r}$. Specifically, each of the orthogonal MIMO links can be represented using Equation 3 by assigning $N=1$. Hence, the capacity of the MIMO DCMC achieving full multiplexing gain for $D$-dimensional, $M$-ary QAM/PSK signals employing $N_{t}$ number of transmit antennas and $N_{r}$ number of receive antennas can be expressed as:

$$
C_{\mathrm{DCMC}}^{\mathrm{MUL}}=\mathcal{N} C_{\mathrm{DCMC}}^{N=1}[\mathrm{bit} / \mathrm{sym}],
$$

where $C_{\text {DCMC }}^{N=1}$ is the capacity of an uncoupled $D$-dimensional DCMC having $N=1$, which can be obtained from Equation 11. Note that the energy of the signal sets is still normalised by $\sqrt{N_{t}}$, when the transmitter does not know the complex Rayleigh-distributed channel coefficient of each of the MIMO links. Similarly, the capacity of the MIMO CCMC achieving full multiplexing gain for $D$-dimensional Gaussian input signals employing $N_{t}$ number of transmit antennas and $N_{r}$ number of receive antennas can be expressed as:

$$
C_{\mathrm{CCMC}}^{\mathrm{MUL}}=\mathcal{N} C_{\mathrm{CCMC}}^{N=1}[\mathrm{bit} / \mathrm{sym}],
$$

where $C_{\mathrm{CCMC}}^{N=1}$ is the capacity of an uncoupled $D$-dimensional CCMC having $N=1$, which can be obtained from Equation 13 and the SNR in Equation 13 is still normalised by $N_{t}$, when the transmitter does not know the channel.

\section{BANDWIDTH EFFICIENCY}

Normalising the channel capacity $C$, which can be represented by either of Equations 11, 13, 14 and 15, with respect to the product of the bandwidth occupied and the signalling period yields another useful performance metric, namely the bandwidth efficiency:

$$
\eta=\frac{C}{W T}=\frac{C}{D / 2}[\mathrm{bit} / \mathrm{s} / \mathrm{Hz}]
$$

The bandwidth efficiency is plotted against the $S N R$ per bit, which is given by: $\frac{E_{b}}{N_{0}}=\frac{S N R}{\eta}$.

\section{NUMERICAL RESULTS}

Figure 2 illustrates the achievable capacity $C$ of both the uncorrelated MIMO Rayleigh-fading channel and that of the AWGN channel for 


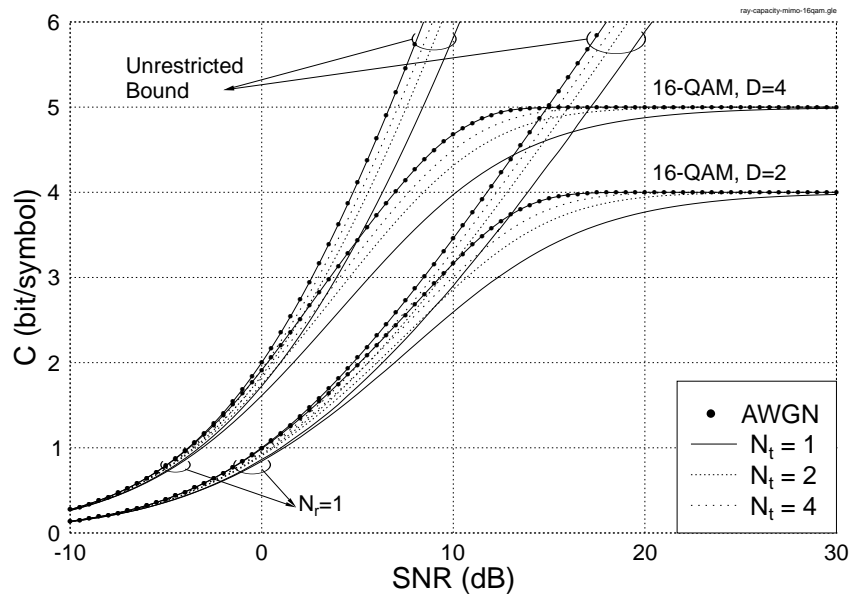

Figure 2: The capacity of the MIMO uncorrelated Rayleigh-fading channel and AWGN channel for 16QAM having $V=1(M=16$, $D=2)$ and $V=2(M=32, D=4)$.

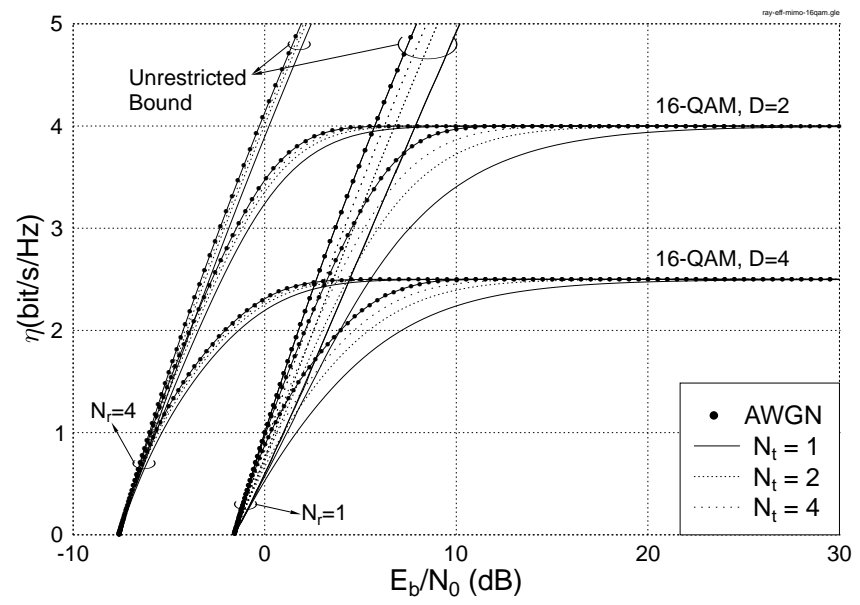

Figure 3: The bandwidth efficiency of the MIMO uncorrelated Rayleigh-fading channel and AWGN channel for 16QAM having $V=1(M=16, D=2)$ and $V=2(M=32, D=4)$.

$L=16$-orthogonal QAM signalling having both $V=1$ and $V=2$, assuming that the transmitter does not know the channel. We denote ' $L=16$-orthogonal QAM having $V=v$ ' as '16QAM, $V=v$ ' for brevity. Again, an $L=16$-orthogonal QAM/PSK signalling having $V=1$ represents classic two-dimensional $L$-ary QAM/PSK signalling. As shown in Figure 2, the achievable capacity of the Rayleigh-fading channel increases as the number of transmit antennas $N_{t}$ increases from 1 to 4 , approaching the capacity of the AWGN channel, which is independent of $N_{t}$.

Figure 3 depicts the bandwidth efficiency $\eta$ of both the uncorrelated MIMO Rayleigh-fading channel and that of the AWGN channel for $L=16$-orthogonal QAM signalling having both $V=1$ and $V=2$, assuming that the transmitter does not know the channel. It is shown in Figure 3 that as $N_{r}$ increases, the bandwidth efficiency of the AWGN channel also improves, hence the corresponding Rayleighfading channel performance follows the same trend. However, the attainable extra transmit diversity gain of the Rayleigh-fading channel reduces, as $N_{r}$ increases, since a near-AWGN performance is achieved by the high-order receiver diversity.
As seen by comparing Figures 2 and 3 for the systems having $N_{r}=1$, the achievable channel capacity increases as the signal dimensionality $D$ increases, although this is attained at a reduced bandwidth efficiency. However, the error-resilience of the power-efficient multi-dimensional orthogonal signals also improves as the dimensionality increases [2]. As evidenced by Figure 3, at low $E_{b} / N_{0}$ the $\eta$ value of 16QAM in conjunction with both $V=1$ and $V=2$ converges to the unrestricted bound. Note that the unrestricted bound is independent of the signal dimensionality.

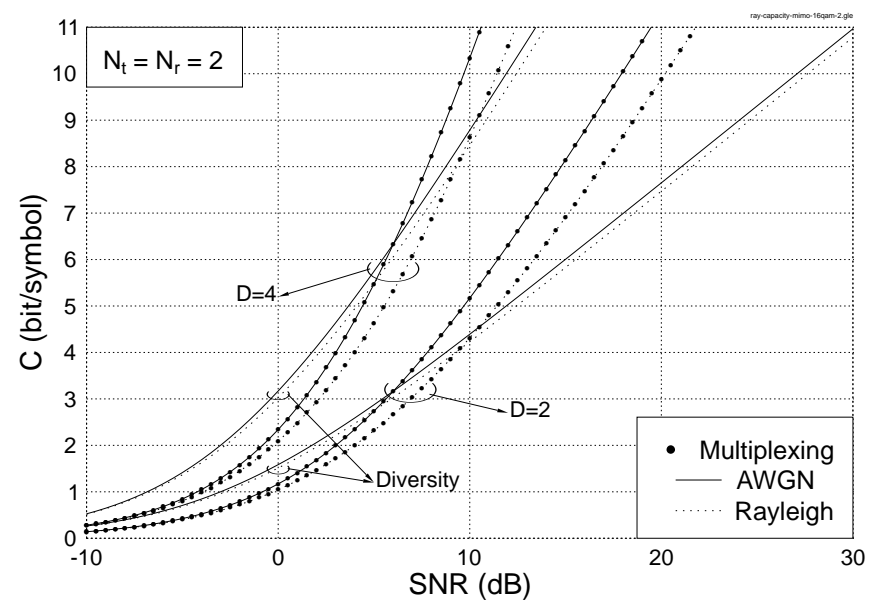

Figure 4: The unrestricted bound of the $D=2$ and 4 dimensional MIMO uncorrelated Rayleigh-fading channel and AWGN channel.

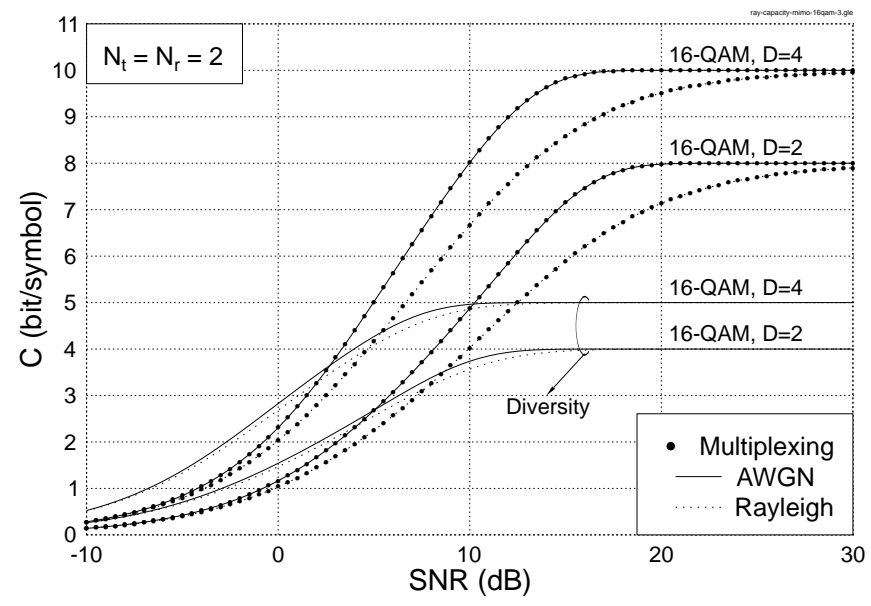

Figure 5: The capacity of the MIMO uncorrelated Rayleigh-fading channel and AWGN channel for 16QAM having $V=1(M=16$, $D=2)$ and $V=2(M=32, D=4)$.

Lets us now compare the achievable capacity of the full-diversity MIMO system to that of the full-multiplexing-gain MIMO system in Figures 4 and 5 using $N_{t}=N_{r}=2$ in the context of $D=2$ and 4 dimensional signalling. Specifically, the capacity of the MIMO CCMC and DCMC are shown in Figures 4 and 5, respectively. Note that the AWGN CCMC capacity for a full-diversity MIMO system characterised in Equation 13 becomes:

$$
C_{\mathrm{CCMC}}^{N}=E\left[\frac{D}{2} \log _{2}\left(1+N_{r} S N R\right)\right],
$$

where the SNR is multiplied by $N_{r}$ since we have $\chi_{2 N}^{2}=\left(N_{r} \times N_{t}\right)$ 
for the AWGN channel. By contrast, the AWGN CCMC capacity of the full-multiplexing-gain MIMO system in Equation 15 becomes:

$$
C_{\mathrm{CCMC}}^{\mathrm{MUL}}=\mathcal{N} E\left[\frac{D}{2} \log _{2}\left(1+\frac{S N R}{N_{t}}\right)\right],
$$

where the expectation value is multiplied by a factor of $\mathcal{N}=N_{t}=$ $N_{r}$, but the SNR is divided by $N_{t}$, since we have $\chi_{2}^{2}=1$ for the AWGN channel. Therefore, at low SNR the full-diversity system outperforms the full-multiplexing-gain system, while the opposite is true at a high SNR, as it is evidenced by Figure 4. As shown in Figure 4, at $\mathrm{SNR}=6 \mathrm{~dB}$ the AWGN capacity curves of the full-diversity system and that of the full-multiplexing-gain system cross over at $C=3.16$ and $6.32 \mathrm{bit} / \mathrm{sym}$, when the signal dimension is $D=2$ and $D=4$, respectively. Similar performance trends are also observed in Figure 4 for the capacity of the Rayleigh fading channel.

Figure 5 shows the capacity curves for the MIMO DCMC employing $D=2$ and $D=4$ dimensional 16QAM signalling. Again, at low SNR (or $C$ ) the full-diversity system outperforms the fullmultiplexing-gain system, while the opposite is true at high SNRs (or $C$ ), as it is evidenced by Figure 5. Explicitly, the full-diversity system outperforms the full-multiplexing-gain system for $C<3.2$ and $C<4 \mathrm{bit} / \mathrm{sym}$ in terms of $D=2$ and $D=4$-dimensional 16QAM signalling, respectively, when communicating over Rayleigh fading channels. Although the full-multiplexing-gain system has a higher asymptotic capacity, the gap between the capacity curves of the Rayleigh fading channel and the AWGN channel is wider in comparison to that of the full-diversity system, as it is shown in Figure 5. Therefore, a full-multiplexing-gain MIMO system provides a higher throughput at the cost of requiring a higher SNR for reliable transmissions. By contrast, a full-diversity MIMO system requires a lower SNR for reliable transmissions at the cost of a lower throughput.

\section{CONCLUSIONS}

Two general formulae, i.e. Equation 11 and 14, were provided for the MIMO channel capacity of multi-dimensional signal sets. Both the channel capacity and bandwidth efficiency of two- and four-dimensiona 16QAM were evaluated, when full diversity gain or full multiplexing gain was achieved. It was shown that transmit diversity is capable of narrowing the gap between the capacity of the Rayleigh-fading channel and the AWGN channel. However, since this gap becomes narrower, when the receiver diversity order is increased, for higher-order receiver diversity the performance advantage of transmit diversity diminishes. The capacity of full-diversity and full-multiplexing-gain MIMO systems was studied, where at a low SNR the full-diversity system has a higher achievable capacity compared to that of the fullmultiplexing-gain system, while the opposite is true at a high SNR. The capacity formulae provided can be used for studying the channel capacity of MIMO systems having various signal dimensionality, different modulation schemes as well as a different number of transmit and receive antennas.

\section{ACKNOWLEDGEMENTS}

The financial support of both the EPSRC, Swindon UK and the EU under the auspices of the Phoenix project is gratefully acknowledged.

\section{REFERENCES}

[1] C. E. Shannon, "A Mathematical Theory of Communication," Bell System Technical Journal, vol. 27, pp. 379-423 and 623-656, June and October 1948
[2] J. G. Proakis, Digital Communications. Mc-Graw Hill International Editions, 3rd ed., 1995.

[3] B. Vucetic and J. Yuan, Space-Time Coding. New York: John WileyIEEE Press, May 2003.

[4] L. Hanzo, T. H. Liew and B. L. Yeap, Turbo Coding, Turbo Equalisation and Space Time Coding for Transmission over Wireless channels. New York, USA: John Willy IEEE Press, 2002.

[5] L. Zheng and D. Tse, "Diversity and Multiplexing: A Fundamental Tradeoff in Multiple Antenna Channels," IEEE Transactions on Information Theory, vol. 49, pp. 1073-1096, May 2003.

[6] V. Tarokh, N. Seshadri and A. R. Calderbank, "Space-time codes for high rate wireless communication: Performance analysis and code construction," IEEE Transactions on Information Theory, vol. 44, pp. 744-765, March 1998.

[7] S. M. Alamouti, "A simple transmitter diversity scheme for wireless communications," IEEE Journal on Selected Areas in Communications, vol. 16, pp. 1451-1458, October 1998.

[8] V. Tarokh, H. Jafarkhani and A.R. Calderbank, "Space-Time Block Codes from Orthogonal Designs," IEEE Transactions on Information Theory, vol. 45, pp. 1456-1467, July 1999.

[9] G. J. Foschini, Jr., "Layered Space-time architecture for wireless communication in a fading environment when using multi-element antennas," Bell Labs Tech. J., pp. 41-59, 1996.

[10] P. E. McIllree, "Channel Capacity Calculations For M-ary NDimensional Signal Sets," M.Eng thesis, The University of South Australia, 1995.

[11] W. C. Lindsey, M. K. Simon, "L-Orthogonal Signal Transmission and Detection," IEEE Transactions on Communications, vol. COM-20, pp. 953-960, October 1972.

[12] J. Wozencraft and I. Jacobs, Principles of communications engineering. New York, USA: John Wiley, 1965.

[13] S.S. Pietrobon, R.H. Deng, A. Lafanechére, G. Ungerböck and D.J. Costello, "Trellis-Coded Multidimensional Phase Modulation," IEEE Transactions on Information Theory, vol. 36, pp. 63-89, January 1990.

[14] S. X. Ng and L. Hanzo, "Space-Time IQ-interleaved TCM and TTCM for AWGN and Rayleigh Fading Channels," IEE Electronics Letters, vol. 38, pp. 1553-1555, November 2002.

[15] L. He and H. Ge, "A New Full-Rate Full-Diversity Orthogonal SpaceTime Block Coding Scheme," IEEE Communications Letters, vol. 7, pp. 590-592, December 2003

[16] R. Gallager, Information Theory and Reliable Communication. John Wiley and Sons, 1968.

[17] G. Ungerböck, "Channel Coding with Multilevel/Phase Signals," IEEE Transactions on Information Theory, vol. 28, pp. 55-67, January 1982. 